\title{
Phyto-nanotechnology:
}

\section{Current status, Challenges, and leads for future}

$\underline{\text { Satya Prakash }}^{\star a}$, Vijay Rani Rajpal $^{\mathrm{b}}$, Samantha Vaishnavic, Renu Deswala

\section{Introduction
Phyto-nanotechnology can address the
challenges of global food security by challenges of global food security
offering Nano-Agri products like biosensors/nanocarriers and by mitigating stress faced under changing climate. However, commercialization of nanotechnology in agriculture struggles despite significant progress at the} \section{application level. \\ Objective 2
Identify possible solutions
for safe and efficient use of
nanotechnology in \\ Objective 2
Identify possible solutions
for safe and efficient use of
nanotechnology in \\ Objective 2
Identify possible solutions
for safe and efficient use of
nanotechnology in \\ Objective 2
Identify possible solutions
for safe and efficient use of
nanotechnology in agriculture.}

\section{Objective 1}

Identify the factors that are delaying commercialization of Nano-based Agri-inputs.

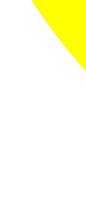
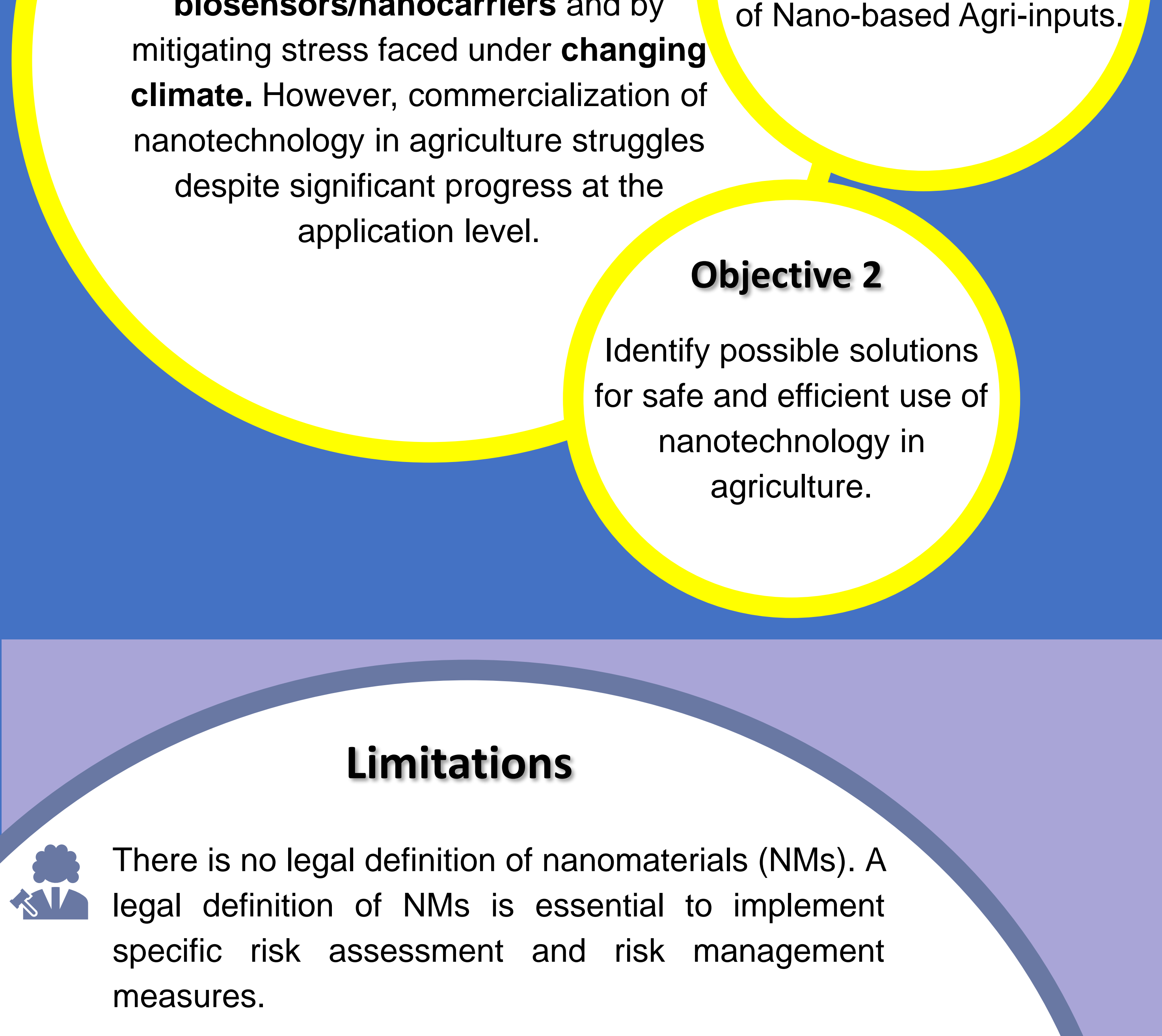
measures.

NP-biomolecule interaction is poorly understood. At our

17. current level of knowledge, it is difficult to predict the potential environmental impacts of nanoparticles.

(10.

There is no uniform or specified legislative framework. Several countries have not issued guidelines for the evaluation of Nano-based Agri-inputs.

Safety assessment studies could not provide a conclusive statement. Therefore, more extensive work is needed.

\section{Methodology}

Latest 150 articles were downloaded from PubMed using search term "Nanotechnology in agriculture".

Abstracts of all the articles were referred to build a literature category graph to identify the key areas currently in focus.

Information from articles related to regulatory aspects and current challenges was gathered to generate a summary of bottlenecks and possible solutions.

\section{Literature Categories}

Green Synthesis

Pathogen/Cancer

Impact assessment

Application

Stress Mitigation

Toxicity concern

Rhizosphere

Omics
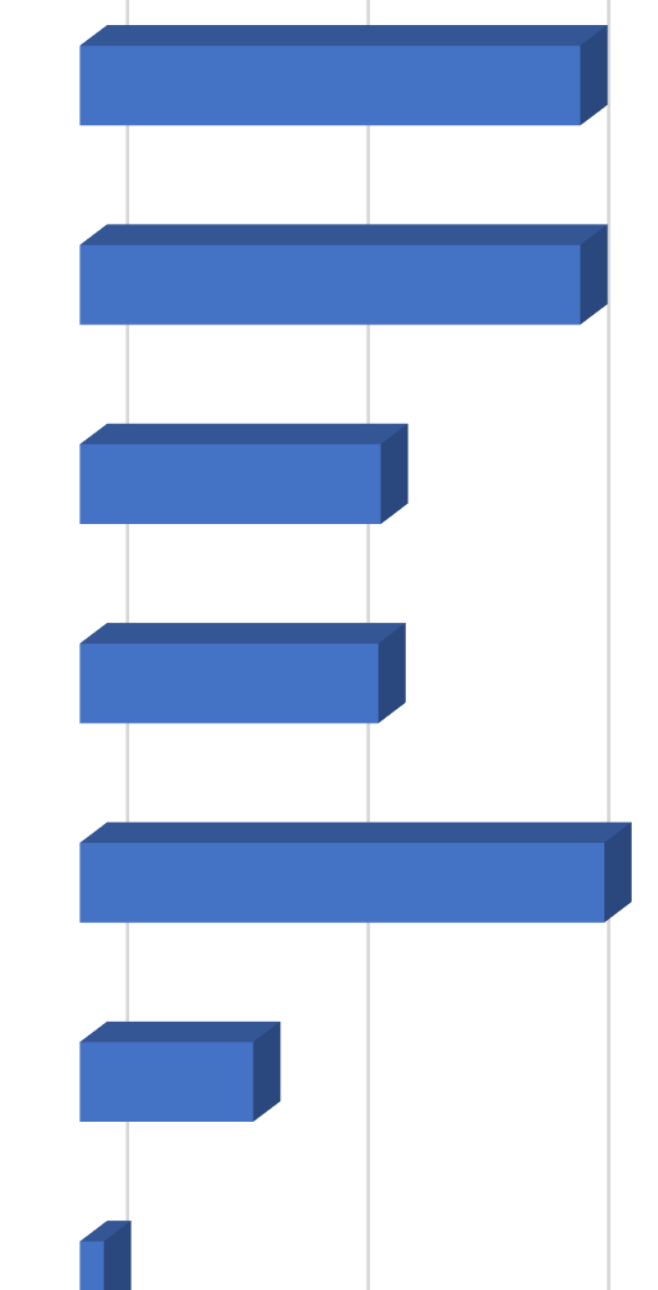

$0 \% \quad 10 \% \quad 20 \% \quad 30 \%$

Nanoparticle-biomolecule interaction?

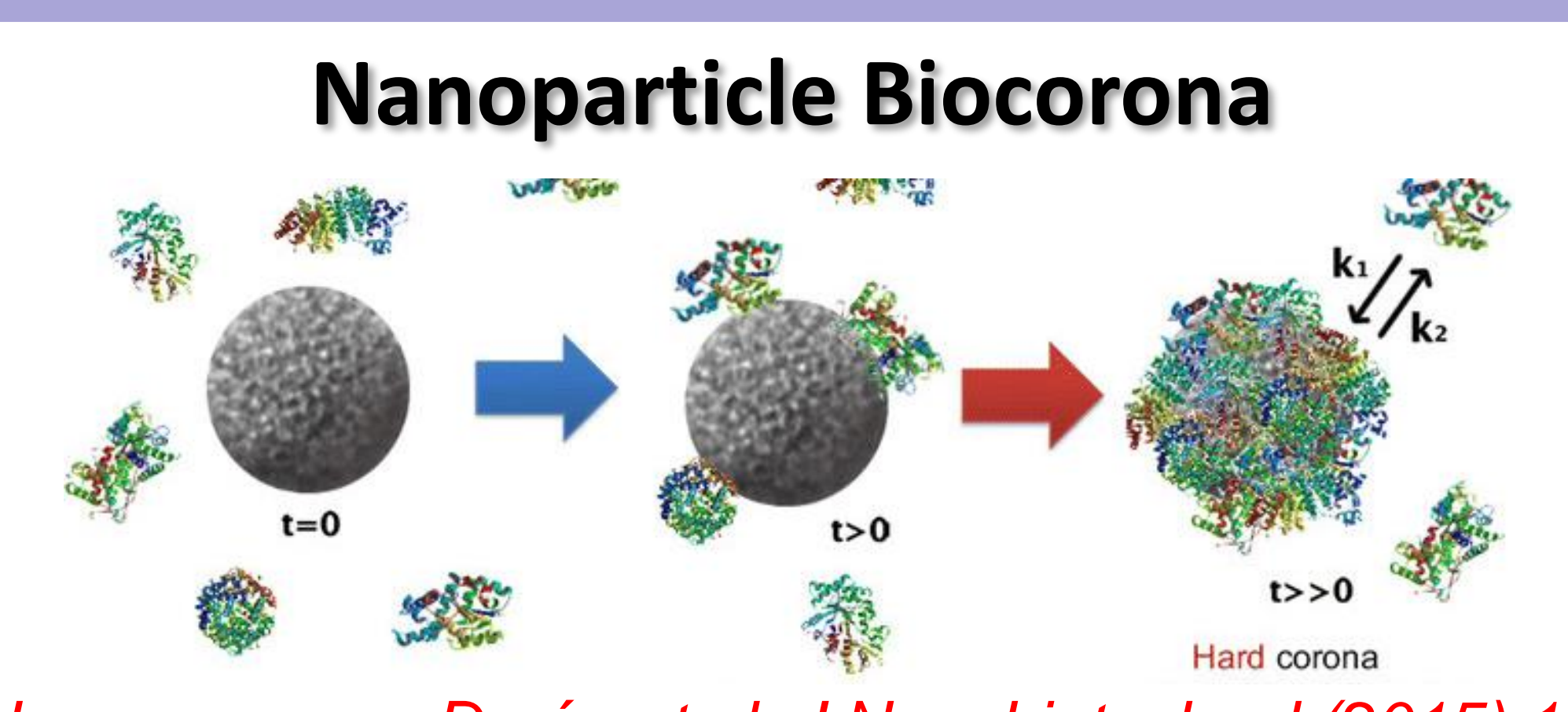

Image source: Durán et al. J Nanobiotechnol (2015) 13:55

$\checkmark$ NP corona formed in plants may be composed of proteins, lipids, carbohydrates and secondary metabolites

$\checkmark$ Composition is influenced by NP Characters and plant specific characters.

$\checkmark$ NP size, stability and surface functionalization changes.

$\checkmark$ Proteins under go change in secondary structure.

$\checkmark$ Potential to alter energy synthesis in Brassica juncea. (AuNP-PC)

Salt tolerance of seedlings in capsicum annum. (MnNPs PC)

\section{Future prospects}

- Definition of NM needs to be addressed.

- More groups should focus on the study of Nano-bio interface.

- Impact assessment studies should also explain underlying mechanisms and laws that determine the outcome of nano-bio integration.

- It is the responsibility of stakeholders to persuade policy makers so that proper legislations can be laid down for the approval of Nano-based Agri-inputs.

- Building of standards and Labs for testing, validation and certification of nano-based agricultural products. 